\title{
Utilizing CDMA Techniques to Improve Ultrasound Based Distance Measurements
}

\author{
Daniel Glaser ${ }^{1}$; Stefan J. Rupitsch ${ }^{1,2}$; Marco Hentschel ${ }^{1}$; Stefan Adelhardt ${ }^{1}$; Reinhard Lerch ${ }^{1}$ \\ ${ }^{1}$ Chair of Sensor Technology, Friedrich-Alexander-University Erlangen-Nuremberg \\ Paul-Gordan-Str. 3-5, 91052 Erlangen, Germany \\ 2 stefan.rupitsch@Ise.eei.uni-erlangen.de
}

\begin{abstract}
Ultrasound based distance measurements are widely used in industrial (e.g., filling level measurements) as well as in automotive applications. In the majority of cases, one or two ultrasound transducers (transceiver) are used to emit and receive the pressure waves. If the speed of sound in the propagation media is known, the distance can easily be calculated by the time-of-flight of the pressure waves. In this contribution, we present ultrasound based distance measurements with the aid of spread spectrum modulation methods, especially so-called Code Division Multiple Access (CDMA) techniques. In particular, Gold sequences are codes for band-spread modulation offering outstanding properties of the auto- and cross-correlation function. Our measurement setup mainly consists of a transducer for emitting pressure waves and a hydrophone for the reception of the pressure waves. The distance between transducer and hydrophone is calculated by the cross-correlation of the excitation signal and output-signal of the hydrophone. The results show that the deviation of measured and prescribed distances are smaller than $0.3 \%$.
\end{abstract}

\section{Introduction}

State of the art in ultrasound distance measurements use short pulses to measure the time interval between start of transmission and reception. Therefore, different approaches are used, depending on the accuracy that is required for a particular application. By simply triggering a specific reception amplitude, the accuracy gets worse for low transmission power and greater distances. Another approach uses correlation to find the peak amplitude by correlating the generated and received signal directly.

Both approaches lack in continuity of the measurement and the possibility to use many transducers in a dense environment with high update rates. In automotive distance control applications, the update rate is of minor importance. But in robotic applications with high speeds, high accelerations and many axes, both, a high update rate and simultaneous measurements in a dense environment are required to detect obstacles. For a pulse echo mode distance measurement, as it is used in ultrasound based level monitors, saturation effects restrain measurements for close distances. This effects arise for instance in water reservoirs with little head space and a high liquid level. Especially by using only a single transducer for pulse echo operation, despreading gain of spread spectrum code modulation makes it possible to lift out the wanted signal. To overcome the single-user limitation, time division multiple access (TDMA), frequency division multiple access (FDMA) and code division multiple access are basic concepts to generate multi-user access to a channel. As already discussed, TDMA reduces the possible update rate, while FDMA is not easy to deploy and even lacks a continuous measurement without special modulation. Here, Code Division Multiplex (CDMA) comes into play, which can be understood as a continuous wave with integrated pulses and many transmission and post-processing parameters qualifying it for different application targets (e.g., low transmission power, multi-user, high resolution, high dynamic, etc.). This paper discusses the use of broad band ultrasound transmission for a simple setup to depict its practicability, not only for more complex scenarios like local positioning [1], [2] or robot applications [3].

The paper is structured mainly in a theoretical part (Sec. 2) that explains the modulation scheme of CDMA and a practical part explaining the experiments (Sec. 3,4) and their results (Sec. 5). Some emphasis in 
theoretical part is put on design decision alternatives and their impact on accuracy. The following sections describe our experimental setup and the measurement procedure including calibration. Experimental results fortify the theoretical considerations by proving the outstanding precision of the measurement results and their advantages compared with other principles. Last section subsumes the results and gives a short insight into planned researches.

\section{Modulation}

\subsection{Digital Modulation}

As stated before and is discussed for positioning systems in [4], the modulation scheme makes use of CDMA spread spectrum techniques to improve the ultrasound based distance measurement. While simple Pseudo-Random Noise (PRN) sequences increase SNR and measurement performance, CDMA with the use of Gold codes (as in GPS) combines many advantages. Gold codes are well selected combinations of m-sequences, which in turn are a subset of PRN sequences with special properties. One possible hardware implementation of a Gold code generator makes use of Linear Feed-back Shift Registers (LFSR) as shown in Fig. 1. The feedback loop is attained with the XOR functions above LFSR 1 and below LFSR 2. The centered XOR combines the taps for code selection (dashed arrows) and feeds it into the output XOR, with its result being used as the phase information $\left(0=0^{\circ}, 1=180^{\circ}\right)$ for carrier modulation. Implying identical LFSR initialization values and feedback positions, diverse tap positions generate different Gold codes fulfilling the orthogonality criterion.

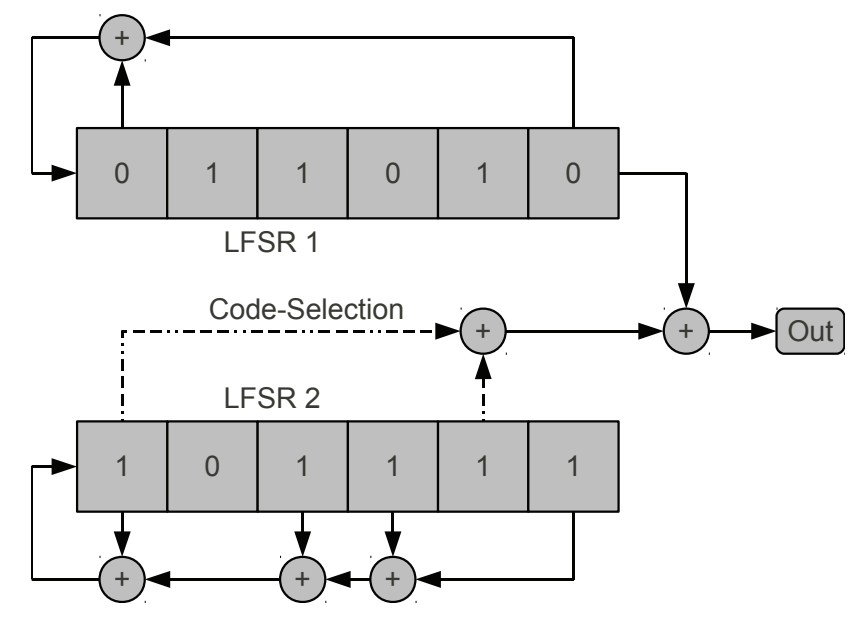

Figure 1: Combination of two linear feedback shift registers that build up the Gold code generator.

While common PRN and m-sequences do not have well defined cross- and auto-correlation characteristics, Gold even defines the correlation values across different codes. These characteristics can be adjusted by the code length itself and the length of the correlation analysis, giving the ability for trade-offs in various applications. The codes are ought to be orthogonal, which means that the cross-correlation has very low maximum values and the auto correlation has a relatively high one, as depicted in Fig. 2. Other codes that promise a higher computation efficiency [5] often relinquish performance in sense of accuracy and configurability compared to Gold codes.

The analysis can also use only part of a full code sequence for correlation to work with reduced SNR, but the length of the transmission sequence should be selected to effectively suppress ambiguities. The full period of the sequence (travel time) depends on the code length $m$ and chip length $l$, usually given as a multiple of the carrier frequency $f$. With a given speed of sound $c$, the distance $d$ of ambiguities can easily be calculated by

$$
d=c \cdot \frac{m l}{f}
$$



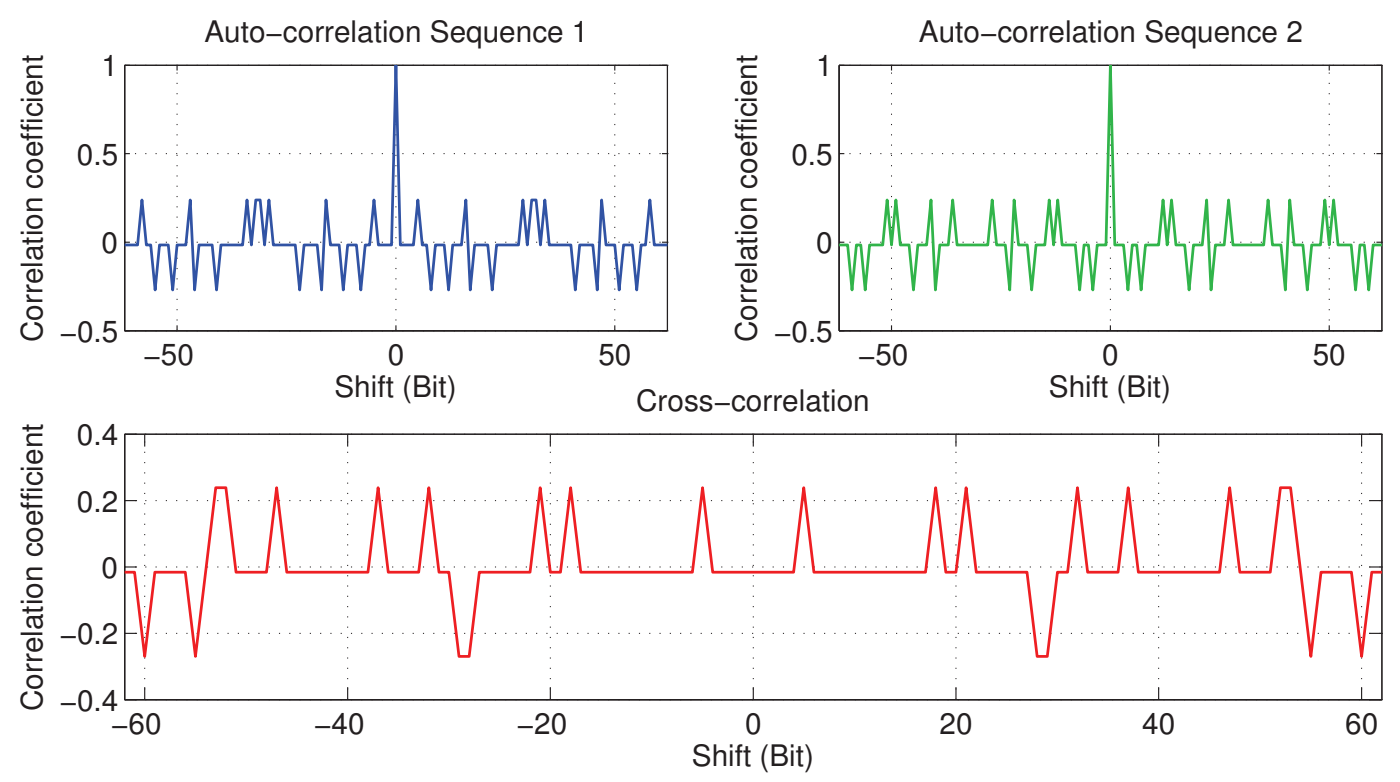

Figure 2: Auto-correlation and cross correlation for two selected gold codes.

For the example used here, the calculation gives an ambiguity distance of

$$
d=1484 \frac{\mathrm{m}}{\mathrm{s}} \cdot \frac{63 \cdot 10}{2.25 \mathrm{MHz}} \approx 0.42 \mathrm{~m}
$$

The choice of chip length directly influences the bandwidth of the spread spectrum signal. The more carrier periods are contained in a single chip, the lower the band-spread and vice versa. The code length mainly defines the robustness to ambiguities.

If only a single measurement unit and the full sequence for correlation is used, the despreading gain for Gold codes is according to the discrete correlation values $\approx 11.4 \mathrm{~dB}$ ([6], codes of length $63: 1,-1 / 63,17 / 63$, $-15 / 63)$. If a continuous distance measurement is presumed, the highest peak can get tracked, resulting in a gain of $\approx 36 \mathrm{~dB}$. Table 1 contains an overview of modulation gains for different code/correlation length and the related ambiguity distances for a a carrier frequency of $2.25 \mathrm{MHz}$ and 10 carrier periods per chip.

\begin{tabular}{|c|c|c|c|c|}
\hline code length & \multicolumn{2}{|c|}{ gain (dB) } & \multicolumn{2}{c|}{$\begin{array}{c}\text { ambiguity } \\
\text { distance (m) }\end{array}$} \\
\cline { 2 - 5 } & overall & peak & water & air \\
\hline 7 & 2.92 & 16.9 & 0.046 & 0.011 \\
\hline 31 & 10,7 & 29.8 & 0.20 & 0.047 \\
\hline $\mathbf{6 3}$ (example) & $\mathbf{1 1 . 4}$ & $\mathbf{3 6 . 0}$ & $\mathbf{0 . 4 2}$ & $\mathbf{0 . 0 9 6}$ \\
\hline 127 & 17.5 & 42.1 & 0.84 & 0.19 \\
\hline 511 & 23.8 & 54.2 & 3.4 & 0.78 \\
\hline 1023 (GPS) & 23.9 & 60.2 & 6.7 & 1.6 \\
\hline 2047 & 30.0 & 66.2 & 13.5 & 3.1 \\
\hline 8191 & 36.0 & 78.2 & 54 & 12.5 \\
\hline
\end{tabular}

Table 1: Performance overview of different Gold code length

\subsection{Analog Modulation}

Different analog carrier modulation schemes can be applied, namely amplitude shift keying (ASK), phase shift keying (PSK) and frequency shift keying (FSK). For CDMA, ASK and PSK are most commonly used. 
They can be substituted by each other in some special cases. The modulation in our example uses PSK with $0^{\circ}$ (' $\left.0^{\prime}\right)$ and $180^{\circ}$ (' 1 '), which can also be described by $-90^{\circ} /+90^{\circ}$ and with ASK when using ' 1 ' and ' -1 ' as the chip symbols. An example use of ASK with symbols ' 0 ' and ' 1 ' ist described in [7].

\section{Measurement Setup}

The measurement setup (Fig. 3(a)) mainly consists of an arbitrary waveform generator (Agilent 3325A), an amplifier (NF Electronic Instruments 4005 High Speed Power Amplifier), a digital oscilloscope (Tektronix TDS 3045B), an ultrasonic transducer (KRAUTKRAMER, $f_{M}=2.25 \mathrm{MHz}$ ), hydrophone (ONDA, $f_{c}=10 \mathrm{MHz}$ ) (see Fig. 3(b)), a water tank with a three axis positioning system carrying the transducer and a PC running MATLAB. MATLAB controls the positioning system, the oscilloscope and the Arbitrary Waveform Generator (AWG), which in turn drives the transducer through the amplifier. The signal that gets measured by the hydrophone is directly fed into one channel and the excitation signal is directly sourcing another channel of the oscilloscope.

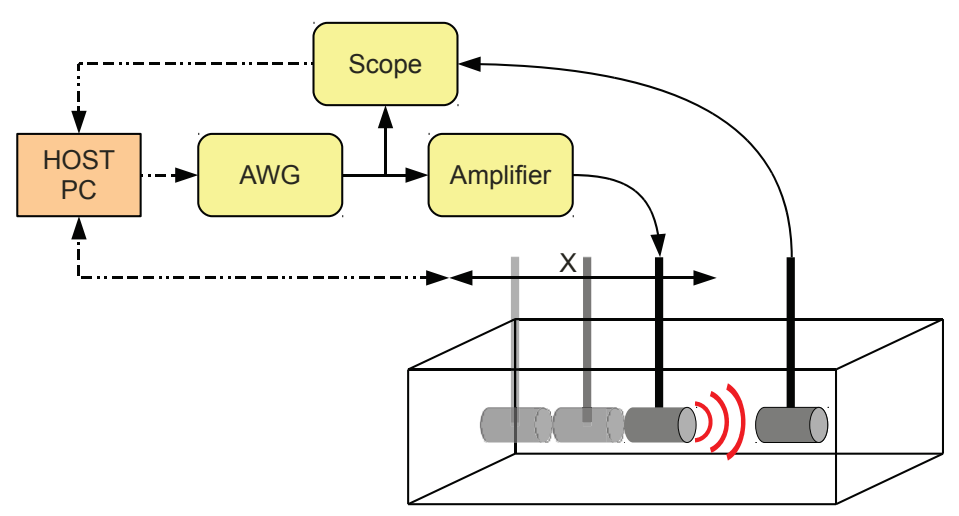

(a)

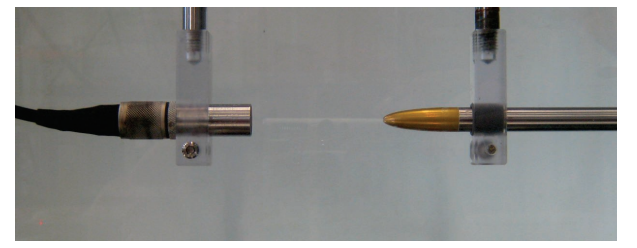

(b)

Figure 3: (a) Measurement setup with instruments, water tank, transducer with positioning axis and hydrophone. Dashed lines: control and data, solid lines: signals (b) Detailed photograph of transducer and hydrophone

\section{Measurement Procedure}

The excitation signal is calculated as described in Sec. 2 and saved to the waveform memory of the AWG. Sampling takes place after the oscilloscope is armed and a trigger is received from the AWG, more or less emulating synchronous operation.

In our first approach the likewise sampled excitation signal is used for correlation with the received signal to extract the time of flight. If not operating synchronously, the time reference needs to be determined by correlating the excitation signal with a synthetically generated signal. A synchronous operation is the appropriate solution for hardware integration e.g., DSP or FPGA. With the current instrument setup only snapshots can be captured, however, the proof of concept can be accomplished.

\subsection{Calibration}

The first measurements are used to calculate the speed of sound in the medium (water from the main), highly depending on temperature and impurities (e.g., concentration of salt). Therefore, two reference points $(100 \mathrm{~mm}$ and $300 \mathrm{~mm}$ ) are destined with well known relative positions and a convenient distance with negligible impact of the near field ( $1 / 2$ " transducer), that is $N=0.25 \cdot D^{2} f c^{-1} \approx 6 \mathrm{~cm}$ [8]. After the calibration sequence, the virtual reference position is calculated, which neglects the near field of the sensor. Some sources of error, which are not subject of this examination, get eliminated by this calibration: 
- Frequency offsets by instrument clocks

- Delays introduced by peripherals

- Temperature and impurity influences

- Absolute errors of the positioning unit

Some errors that can not be eliminated by calibration:

- Clock jitter of the instruments

- Electric and acoustic noise from the environment

- Relative errors (repeat accuracy) of the positioning unit

\section{Experimental Results}

To investigate the potential accuracy of the CDMA method itself, we performed a set of 10 tests for each position from 100 to $300 \mathrm{~mm}$ with an increment of $3 \mathrm{~mm}$. Figure 4(a) shows the correlation function as a function of position and sample time. We used $100 \mathrm{mV}$ of amplitude and $2.25 \mathrm{MHz}$ of carrier frequency. After receiving the hydrophone signal with the digital scope, correlation and application of an envelope function takes place.

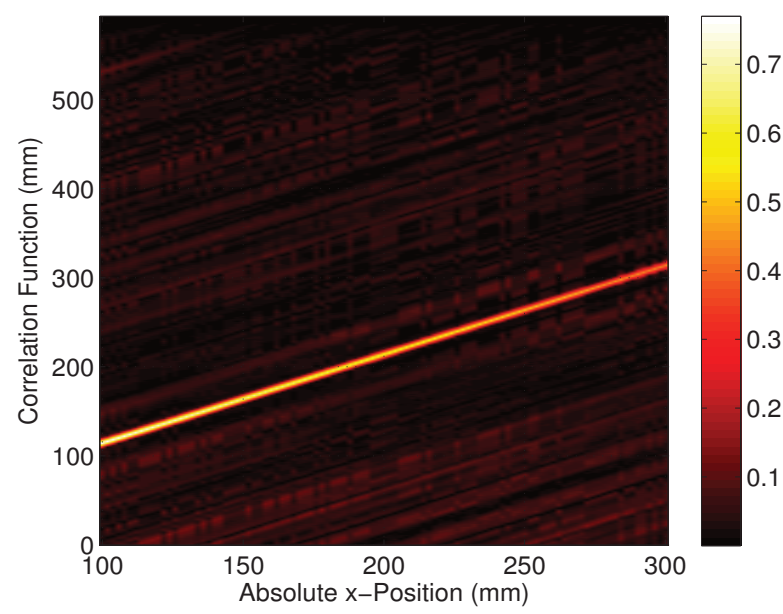

(a)

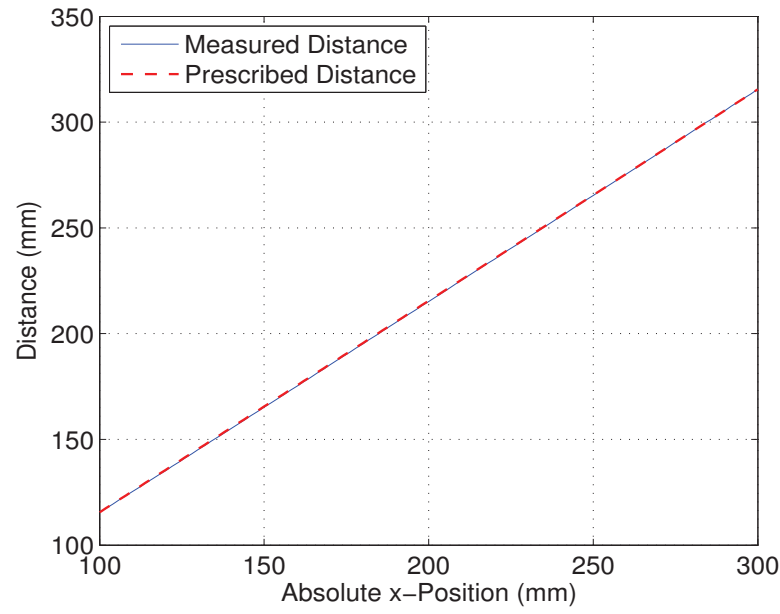

(b)

Figure 4: (a) The landscape of the correlation function with respect to the x-position. (b) The maximum value extracted from the left diagram matches the distance.

Figure 4(b) compares the distances of the positioning unit (red dashed line) and the distances measured with ultrasound. Figure 5(a) in turn shows the error of the measurement in percentage of the absolute position while figure $5(\mathrm{~b})$ shows the error as a function of excitation amplitude at a single position. Since static errors from positioning unit and variation in speed of sound are already covered by the calibration procedure, the data show mainly the pure performance of the measurement approach, still including some correctable deviations, owing to the carrier and disparity of near and far field. It is notable that even with extremely low excitation signals, the distance is quite accurate, still improvable by using longer Gold codes.

\section{Conclusion and Outlook}

Experiments have shown that CDMA modulation with Gold codes has many advantages for ultrasound distance measurement. It improves signal-to-noise ratio by magnitudes, allows separation of signal sources and improves accuracy. The improvement in SNR allows to reduce excitation power while still being able 


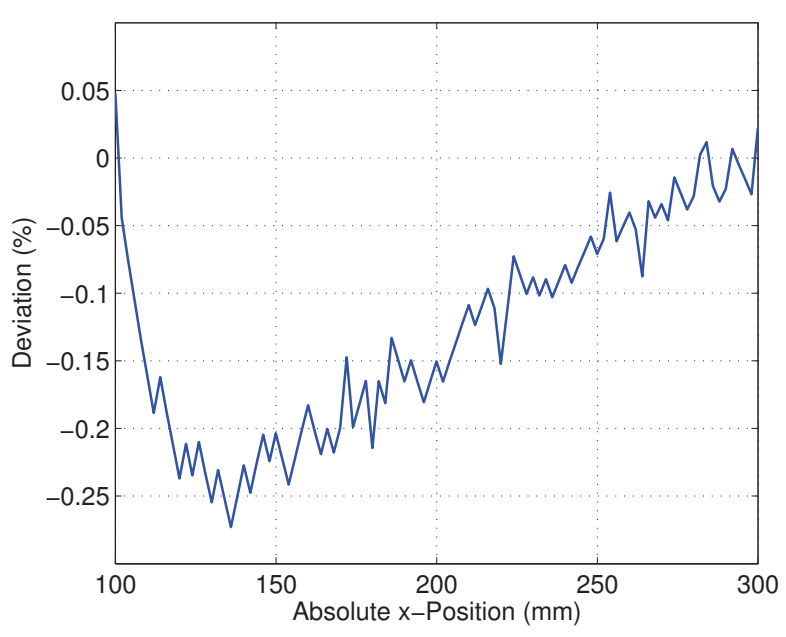

(a)

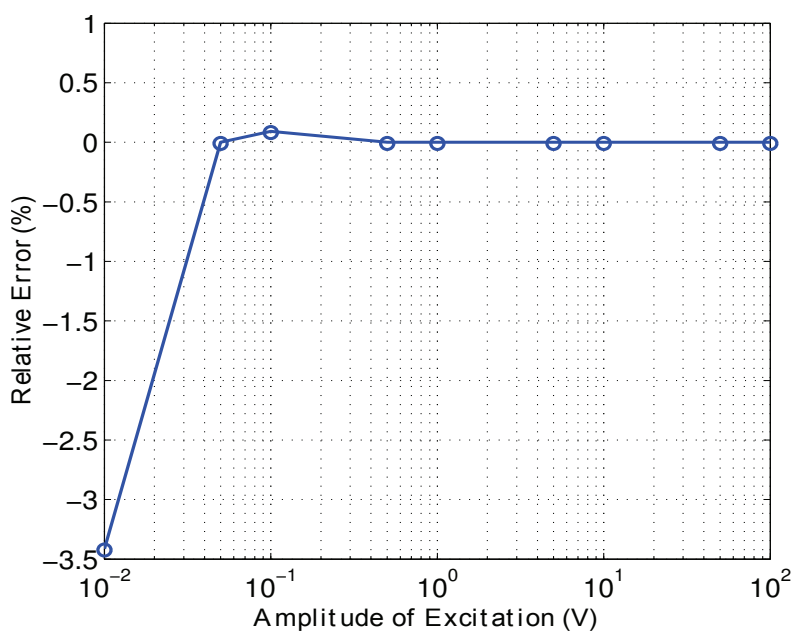

(b)

Figure 5: (a) Deviation of the measured distance to the positioning unit position. (b) Measurement error at a low distance with variation of the excitation amplitude.

to track the distance continuously. The increase in gain can also be utilized to decrease the resolution of the AD converter of the analog frontend, even down to a single bit. However, it must be mentioned that the required processing power is rather high and to track a single signal continuously makes DSP or FPGA indispensable. Being able to track a multiple of signal sources at once gives the opportunity to make use of adjacent devices by asynchronously tracking their signals.

Our future investigations will firstly concentrate on power consumption analysis of such a system, pulse echo mode measurement and on various ultrasound applications beside distance measurement. We also intend to implement the approach in FPGAs, allowing to run tests even with very long codes, which was not possible due to the very limited sample space and the low arbitrary waveform memory of our instruments. With hardware integrated correlation, it will be possible to track signals in real-time without instrument limitations. Improvements in detecting very low signals with removing known candidates by post-processing are also planned for the future.

\section{References}

[1] J. Prieto, A. Jimenez, and J. Guevara, "Subcentimeter-accuracy localization through broadband acoustic transducers," in Intelligent Signal Processing, 2007. WISP 2007. IEEE International Symposium on, October 2007, pp. 1 $-6$.

[2] F. Granja and A. Ruiz, "Ultrasound modulation and codification for localization systems," Instituto de Automatica Industrial (CSIC), Madrid, Spain, 2006.

[3] G. Hueber, T. Ostermann, T. Bauernfeind, R. Raschhofer, and R. Hagelauer, "New approach of ultrasonic distance measurement technique in robot applications," in Signal Processing Proceedings, 2000. WCCC-ICSP 2000. 5th International Conference on, vol. 3, 2000, pp. 2066 -2069 vol.3.

[4] M. Perez, J. Urea, A. Hernandez, A. Jimenez, D. Ruiz, F. Alvarez, and C. De Marziani, "Performance comparison of different codes in an ultrasonic positioning system using ds-cdma," in Intelligent Signal Processing, 2009. WISP 2009. IEEE International Symposium on, August 2009, pp. $125-130$.

[5] A. Hernández, J. Urena, D. Hernanz, J. Garcia, M. Mazo, J. Dérutin, J. Serot, and S. Palazuelos, "Real-time implementation of an efficient Golay correlator (EGC) applied to ultrasonic sensorial systems," Microprocessors and Microsystems, vol. 27, no. 8, p. 397, 2003.

[6] E. Dinan and B. Jabbari, "Spreading codes for direct sequence cdma and wideband cdma cellular networks," Communications Magazine, IEEE, vol. 36, no. 9, pp. 48 -54, September 1998.

[7] L. Wu, Y. Zeng, and H. He, "Novel ultrasonic ranging approach based on spread spectrum modulation," in Communication Technology, 2006. ICCT '06. International Conference on, November 2006, pp. 1 -4.

[8] R. Lerch, G. Sessler, and D. Wolf, Technische Akustik: Grundlagen und Anwendungen. S Springer, 2009. 